\title{
The Relationship between Moral Hazard and Insurance Fraud ${ }^{+}$
}

\author{
Mahito Okura \\ Associate Professor, Faculty of Economics, Nagasaki University \\ Address: 4-2-1 Katafuchi, Nagasaki-city, Nagasaki 850-8506, JAPAN \\ Email: okura@nagasaki-u.ac.jp.
}

\section{Purpose:}

The purpose of this research is to investigate the insurance market in which moral hazard and insurance fraud coexist. In this situation, this research examines the relationship between moral hazard and insurance fraud. Also, this research shows how the amount of policyholder's effort to lower accident probability changes when insurance firm increases their investment in preventing insurance fraud.

\section{Design/methodologylapproach:}

Using a theoretical model containing five-stages, we shed light on how the possibility of insurance fraud affects the amount of policyholder's effort.

\section{Findings:}

The main results of this research are as follows. First, the amount of policyholder's effort is a weakly monotone decreasing function of the insurance firm's investment in preventing insurance fraud. Second, unlike in previous moral hazard models, policyholder chooses a strictly positive amount of effort even in the full insurance case because the possibility of insurance fraud gives an incentive to realize policyholder's effort. Third, the amount of insurance firm's investment in preventing insurance fraud depends on whether it wants to give an additional incentive to policyholder's effort in exchange for realizing the possibility of insurance fraud.

\section{Originality/value:}

This research is the first paper to investigate the relationship between moral hazard and insurance fraud by using the microeconomic theory.

\footnotetext{
+ The author wishes to thank the participants at Working Group on Globalization of Insurance Research sponsored by The Dai-ichi Life Insurance Company, Limited and Dai-ichi Life Research Institute Inc., 2012 Spring Meeting of Japan Association for Applied Economics, and 16th Asia-Pacific Risk and Insurance Association Annual Meeting for their comments. The author also would like to acknowledge financial support by the Ministry of Education, Culture, Sports, Science and Technology in the form of a Grant-in-Aid for Young Scientists (B), 21730339 and 24730362.
} 


\section{Keywords:}

Moral Hazard, Insurance Fraud, Insurance Market

\section{Paper type:}

Research Paper

\section{Introduction}

Although purchasing insurance can reduce and allocate individuals' risks more efficiently, providing insurance generates costs. Thus, even if insurance is a very effective way to manage individuals' risks, they may not choose full insurance. In the extreme case, individuals may choose not to purchase any insurance at all because the costs are too high ${ }^{1}$. That makes analysis of these costs an important issue in insurance studies. It is well known that insurance firms incur additional costs after their policyholders purchase their insurance because of the existence of information-deficiencies. These additional costs relate to moral hazard and insurance fraud. Moral hazard analysis relates to policyholders' amount of effort to lower the accident probabilities. An important aim in moral hazard studies is to assess changing attitudes toward risks after purchasing insurance because excessive insurance coverage leads to a diminished incentive to lower accident probability. Insurance fraud arises when insurance firms, unlike their policyholders, are unable to determine whether claimed accidents really occurred. In that situation, policyholders may have an incentive to claim on their insurance policy even if accidents did not occur. ${ }^{2}$ In practice, insurance firms adopt monitoring systems to prevent moral hazard and insurance fraud. However, even if such systems work well, these systems per se generate costs.

The difference between moral hazard and insurance fraud is explained in some insurance textbooks. According to Dorfman (2008), insurance fraud in our research indicates "moral hazard" and moral hazard in our research indicates "morale hazard", respectively. This classification may be based on the background if the person who commits moral hazard is "abnormal". Another kind of explanation about the difference is given from informationdeficiencies. According to Powers (2012, Chapter 8), moral hazard is occurred in the case of unobservable information, which means neither insurance firms nor policyholders know the information about some factors and so it is in the case of symmetric information. In contrast, insurance fraud is occurred in the case of asymmetric information, which shows policyholders

\footnotetext{
1 Harrington and Niehaus (2003) points out these costs limit the insurability of risk.

2 More details about the concept of moral hazard from its history are found in, for example, see Baker (1996) and Rowell and Connelly (2012).
} 
possess their private information. ${ }^{3}$

Many researchers in the fields of both insurance and microeconomics studies have shed light on the characteristics of (insurance) markets under moral hazard and insurance fraud. Moral hazard studies include Arrow (1963, 1968), Pauly (1968, 1974), Holmstrom (1979), Shavell (1979a). Moreover, contract theory, which includes principal-agent models, also treats moral hazard problems such as Shavell (1979b), Grossman and Hart (1983), Holmstrom and Milgrom (1987, 1991), and Kojima and Okura (2008). Economic researches into mechanisms of insurance fraud include Picard (1996), Boyer (2000a, 2000b, 2007) and Dionne et al. (2009). However, many previous studies tended to deal exclusively with either moral hazard or insurance fraud, but not both. Thus, the relationship between moral hazard and insurance fraud is unclear. More specifically, we cannot know how the amount of policyholder's effort changes when insurance firm increases their investment in preventing insurance fraud. The purpose of this research is to investigate that issue and examine the relationship between moral hazard and insurance fraud.

The article is organized as follows. Section 2 develops a model that includes both moral hazard and insurance fraud. Section 3 derives the equilibrium of the model. Concluding remarks are in Section 4.

\section{The Model}

Suppose that there are one risk-averse policyholder and one risk-neutral insurance firm. This policyholder has initial wealth before purchasing insurance denoted by $W>0$. The policyholder has already purchased insurance whose premium is $\alpha p$ and the insurance money is $\alpha D$ where $p>0$ represents the amount of insurance premium in the case of full insurance, $D>p$ is the amount of damage, and $0<\alpha \leq 1$ is the insurance coverage rate, respectively. The following five-stage game is considered.

In the first stage, the insurance firm decides the amount of the investment in preventing insurance fraud. Assume that this investment is irreversible. Thus, the insurance firm cannot recoup that investment cost even if insurance fraud does not occur. $r$ represents the amount of this investment and the probability that the insurance firm prevents insurance fraud. The

\footnotetext{
3 In Powers (2012, Chapter 8), there are two kinds of insurance fraud, "planned fraud" and "opportunistic fraud". The former is that a policyholder pretends that the insured item has to be stolen. The latter is that after the accident occurs, a policyholder pads the bill to receive more insurance money when a policyholder claims. In this research, we will only focus on planned fraud because we will consider the situation in which insurance firms cannot know whether the accident occurs.
} 
investment cost function is assumed to be specified by $(1 / 2) r^{2}$.

In the second stage, after observing the amount of that investment, policyholder decides their own amount of effort for lowering the accident probability. $e$ represents the amount of effort and the probability that the accident does not occur. The effort cost is assumed to be separable and specified by $(1 / 2) e^{2}$.

In the third stage, whether the accident occurs is decided in accordance with the accident probability that was decided in the second stage. If the accident occurs, the policyholder naturally claims and receives its insurance money from the insurance firm, and the game ends. Alternatively, if the accident does not occur, the game proceeds to the fourth stage.

In the fourth stage, the policyholder can choose whether to claim the insurance money. If the policyholder does not claim, that is, the policyholder is honest, no problems arise and the game ends at this stage. In contrast, if the policyholder claims, that is, the policyholder is dishonest, the game proceeds to the fifth stage.

In the fifth stage, whether the policyholder receives insurance money is decided in accordance with the probability calculated in the first stage. If policyholder succeeds in the insurance fraud, policyholder receives the insurance money $\alpha D$. In contrast, policyholder fails in the insurance fraud, the claim is rejected and the policyholder becomes liable to a penalty denoted by $x \geq 0$. We assume that that amount of penalty is not related to the insurance payoff such as fine to the government.

Before solving the above five-stage game, to simplify the expressions, the following variables are introduced

$$
\begin{aligned}
& u_{A} \equiv u(W-\alpha p-D+\alpha D) \\
& u_{N N} \equiv u(W-\alpha p) \\
& u_{N S} \equiv u(W-\alpha p+\alpha D) \\
& u_{N F} \equiv u(W-\alpha p-x)
\end{aligned}
$$

where $u(\bullet)$ represents the policyholder's utility function and assumes that $u^{\prime}(\bullet)>0$ and $u^{\prime \prime}(\bullet) \leq 0 . u_{A}, u_{N N}, u_{N S}$, and $u_{N F}$ represent the policyholder's utility when the accident occurs, when the accident does not occur and the policyholder does not claim, when the accident does not occur and the policyholder's insurance fraud succeeds, and when the accident does not 
occur and the policyholder's insurance fraud fails, respectively. Also, we easily find that $u_{N S}>u_{N N} \geq u_{A}, u_{N F}$.

\section{Deriving the Equilibrium}

Because the decisions of either the policyholder or the insurance firm are in the first, second, and fourth stages, these three stages are analyzed by backward induction.

First, consider the fourth stage. In this stage, the policyholder decides whether to claim insurance money in the case where the accident does not occur. If the policyholder claims, its expected utility is $r u_{N F}+(1-r) u_{N S}$. In contrast, if the policyholder does not claim, its expected utility is $u_{N N}$. Thus, the condition in which the policyholder chooses to claim can be written as

$$
r u_{N F}+(1-r) u_{N S} \geq u_{N N} \Rightarrow r \leq \frac{u_{N S}-u_{N N}}{u_{N S}-u_{N F}} .
$$

Equation (1) implies that the policyholder tends to claim when the insurance firm's amount of the investment is relatively small. Denote that $\hat{r} \equiv\left(u_{N S}-u_{N N}\right) /\left(u_{N S}-u_{N F}\right)$ and we can easily prove $0<\hat{r} \leq 1$ because $u_{N N} \geq u_{N F}, u_{N S}>u_{N N}$, and $u_{N S}>u_{N F}$.

Next, consider the second stage. This stage can be divided into the "fraud case," which is indicated by $r \leq \hat{r}$, and the "no-fraud case," which is indicated by $r \geq \hat{r}$.

In the fraud case, the policyholder's expected utility, which is denoted by $\tilde{U}$, can be written as

$$
\tilde{U}=(1-e) u_{A}+e\left\{r u_{N F}+(1-r) u_{N S}\right\}-\frac{1}{2} e^{2} .
$$

Then, the optimal amount of effort can be computed as follows

$$
\frac{\partial \tilde{U}}{\partial e}=0 \Rightarrow \tilde{e}^{*}=r u_{N F}+(1-r) u_{N S}-u_{A}
$$

Also, we find

$$
\frac{\partial \tilde{e}^{*}}{\partial r}=u_{N F}-u_{N S}<0
$$


Equation (4) indicates that the optimal amount of effort increases when the amount of investment decreases. In other words, the optimal amount of effort is a strictly monotone decreasing function of the amount of investment. Furthermore, because $\tilde{e}^{*}=u_{N S}-u_{A}$ in the case of $r=0$, and $\tilde{e}^{*}=u_{N N}-u_{A}$ in the case of $r=\hat{r}$, we know that $u_{N N}-u_{A} \leq \tilde{e}^{*}<u_{N S}-u_{A}$. To guarantee the inner solution of $\tilde{e}^{*}$, we assume that $u_{N S}-u_{A} \leq 1$.

In contrast, the policyholder's expected utility in the no-fraud case, which is denoted by $U$, can be written as

$$
U=(1-e) u_{A}+e u_{N N}-\frac{1}{2} e^{2} .
$$

Then, the optimal amount of effort can be computed as follows

$$
\frac{\partial U}{\partial e}=0 \Rightarrow e^{*}=u_{N N}-u_{A}
$$

Because insurance fraud is not realized in the later stage, the optimal amount of effort in equation (6) is independent of the amount of the investment. Also, because $\tilde{e}^{*} \geq u_{N N}-u_{A}$, we know $\tilde{e}^{*} \geq e^{*}$.

Using the above discussion, we show the relationship between the amounts of effort and investment in Figure 1. 
Figure 1: The relationship between the amounts of effort and investment

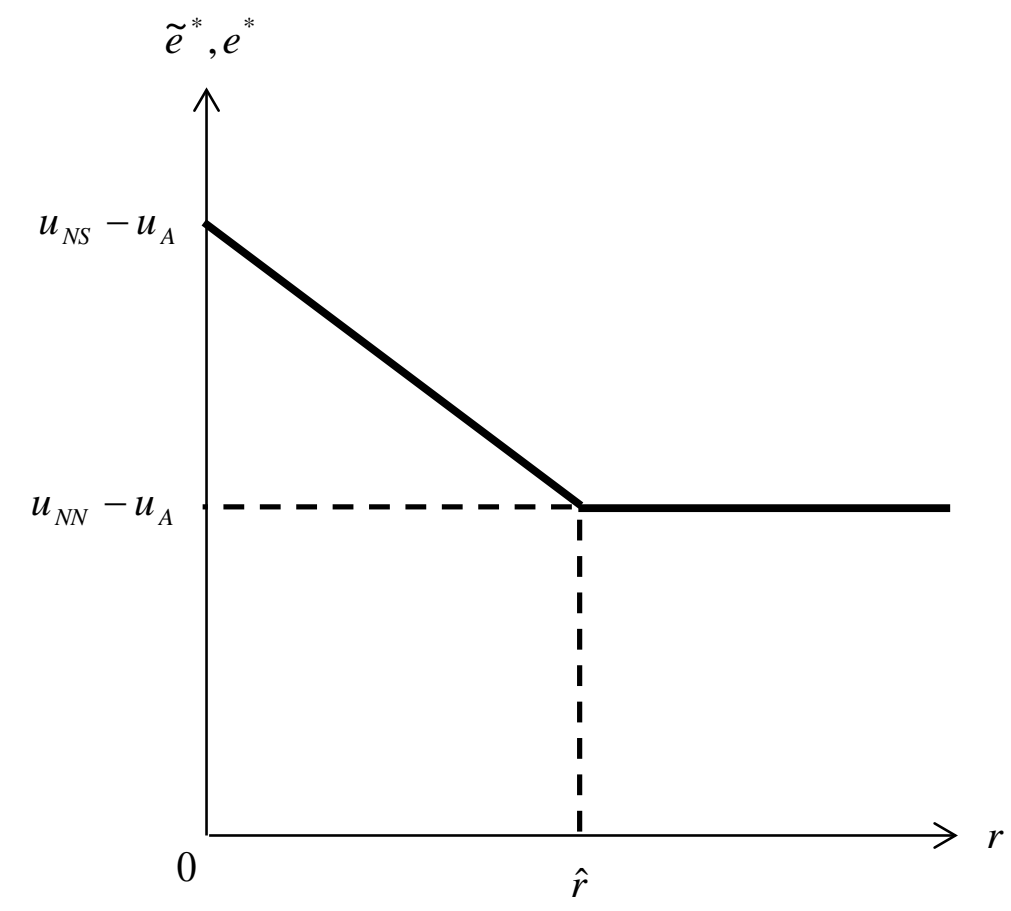

From Figure 1, we find that the policyholder's amount of effort for lowering accident probability is a weakly monotone decreasing function of the insurance firm's amount of investment in preventing insurance fraud. This implies that insurance firm's investment in preventing insurance fraud may lower policyholder's amount of effort. It also suggests why insurance firms do not choose perfect monitoring to prevent insurance fraud.

Last, we consider the first stage where we compute the optimal amount of investment in the fraud and no-fraud cases. Then, we derive the equilibrium through comparison of insurance firm's expected payoffs in the fraud and no-fraud cases.

In the no-fraud case, the insurance firm chooses the lowest amount of investment because the policyholder's optimal amount of effort is decided regardless of the amount of that investment. Thus, the insurance firm surely chooses $r=\hat{r}$. Then, the insurance firm's expected payoff, which is denoted by $\Pi$, can be written as

$$
\Pi=\left(1-e^{*}\right)(\alpha p-\alpha D)+e^{*} \alpha p-\frac{1}{2} \hat{r}^{2}
$$

In contrast, in the fraud case, increasing the amount of investment has both the advantage of 
lowering accident probability and the disadvantage of raising investment cost. Also, it may lead to change in the probability of paying out fraudulent insurance claims which is indicated by $e(1-r)$. The insurance firm's expected payoff, which is denoted by $\tilde{\Pi}$, can be written as

$$
\tilde{\Pi}=\left(1-\tilde{e}^{*}\right)(\alpha p-\alpha D)+\tilde{e}^{*}\{r \alpha p+(1-r)(\alpha p-\alpha D)\}-\frac{1}{2} r^{2}
$$

Then, the optimal amount of investment in the range $r \leq \hat{r}$ can be computed as

$$
\frac{\partial \tilde{\Pi}}{\partial r}=0 \Rightarrow \tilde{r}^{*}=\frac{\alpha D\left(u_{N S}-u_{A}\right)}{1+2 \alpha D\left(u_{N S}-u_{N F}\right)}
$$

To guarantee the inner solution of equation (9), the following inequality is assumed to be satisfied $^{4}$

$$
u_{N S}-u_{N N}+\alpha D\left(u_{N S}-u_{N F}\right)\left(u_{A}+u_{N S}-2 u_{N N}\right)>0 .
$$

In the no-fraud case, the insurance firm has the following two options to choose the amount of investment. The first option is to choose the relatively low amount of investment denoted by $\tilde{r}^{*}$. In this case, although insurance fraud occurs, a relatively high amount of effort can be realized and investment cost becomes relatively cheap. The second option is to choose the relatively high amount of investment denoted by $\hat{r}$. In this case, although the amount of effort becomes relatively low and investment cost becomes relatively expensive, insurance fraud does not occur.

To compare the two options, equations (7) and (8) are transformed as follows.

$$
\begin{aligned}
& \Pi=\alpha p-\left(1-e^{*}\right) \alpha D-\frac{1}{2} \hat{r}^{2}, \\
& \tilde{\Pi}=\alpha p-\left(1-\tilde{e}^{*} \tilde{r}^{*}\right) \alpha D-\frac{1}{2} \tilde{r}^{*^{2}} .
\end{aligned}
$$

Because $\tilde{r}^{*} \leq r^{*}, \tilde{e}^{*} \tilde{r}^{*} \geq e^{*}$ is the sufficient condition to choose $\tilde{r}^{*}$. In order to know the implications of this sufficient condition, $\tilde{e}^{*} \tilde{r}^{*} \geq e^{*}$ is transformed as follows

$$
\left(1-\tilde{e}^{*}\right)+\tilde{e}^{*}\left(1-\tilde{r}^{*}\right) \leq 1-e^{*}
$$

\footnotetext{
${ }^{4}$ If equation (10) is not satisfied, the corner solution $\tilde{r}^{*}=\hat{r}$ is realized.
} 
Both sides of equation (13) represent the probabilities of paying insurance money in the fraud and no-fraud cases. Thus, equation (13) implies the insurance firm chooses $\tilde{r}^{*}$ when the probability of paying insurance money in the fraud case is smaller than that in the no-fraud case.

To confirm the possibility of satisfying $\tilde{e}^{*} \tilde{r}^{*} \geq e^{*}$, we define $f \equiv \tilde{e}^{*} \tilde{r}^{*}-e^{*}$ and compute it as follows

$$
f=\frac{\alpha D\left(u_{N S}-u_{A}\right)^{2}\left\{1+\alpha D\left(u_{N S}-u_{N F}\right)\right\}}{\left\{1+2 \alpha D\left(u_{N S}-u_{N F}\right)\right\}^{2}}-\left(u_{N N}-u_{A}\right) .
$$

If $f \geq 0$, the insurance firm chooses $\tilde{r}^{*}$. However, we cannot derive a general result from equation (14). Thus, which option the insurance firm chooses is ambiguous. In other words, it is possible that the relatively low amount of investment that realizes policyholder's insurance fraud may become the equilibrium.

To shed light on some characteristics of the equilibrium, the following two special cases are investigated. ${ }^{5}$

The first special case is $\alpha=1$ which represents full insurance. Then, $f \geq 0$ is always satisfied because $u_{N N}=u_{A}$. From this confirmation, we find that the insurance firm chooses a relatively low amount of investment when policyholder has full insurance. The logic driving this result is as follows. Because of the full insurance, the policyholder does not have an incentive to lower their accident probability if there is no possibility of achieving insurance fraud. However, if insurance firm chooses a relatively low amount of investment, the possibility of insurance fraud is realized and it derives policyholder's effort. Even if insurance fraud occurs, insurance firm becomes more profitable because expected insurance money becomes less.

Also, substituting $r=\tilde{r}^{*}$ and $\alpha=1$ into equation (3), we have

$$
\tilde{e}^{*}=\frac{\left(u_{N S}-u_{A}\right)\left\{D\left(u_{N S}-u_{N F}\right)+1\right\}}{2 D\left(u_{N S}-u_{N F}\right)+1}>0 .
$$

Equation (15) implies that the policyholder chooses a strictly positive amount of effort even if

\footnotetext{
5 The following two special cases are always satisfied the equation (10).
} 
$\alpha=1$. According to previous moral hazard models, the policyholder never chooses to make an effort in the case of $\alpha=1$ because policyholder's wealth is not changed even if an accident occurs. From that viewpoint, a strictly positive amount of effort in the case of $\alpha=1$ seems to be counterintuitive. However, in our model containing in insurance fraud, the policyholder has another incentive to realize no-accident situation because the possibility of insurance fraud gives an incentive to realize policyholder's effort.

The second special case is $\alpha \approx 0$ which represents a very low insurance coverage rate. In this special case, $f<0$ is realized because the first term of the right-hand side in equation (14) is almost zero while the second term is negative. Also, from the definition of $\hat{r}$ and equation (5), the both amount of investments in the fraud and no-fraud cases are almost zero because $u_{N S} \approx u_{N N}$ and $\alpha \approx 0$. Thus, insurance firm chooses relatively high amount of investment when the insurance coverage rate is very low. The logic driving this result is as follows. Because the insurance coverage rate is low, the policyholder already has an incentive to avoid accidents even if there is no possibility of realizing insurance fraud, while the insurance firm wants to prevent insurance fraud without providing additional incentives for policyholder's effort.

From the discussion of the two special cases, we find that which options the insurance firm chooses depends on whether the insurance firm wants to give an additional incentive to policyholder's effort in exchange for realizing the possibility of insurance fraud. Also, this result shows that moral hazard and insurance fraud have a close interlinked relationship.

\section{Concluding Remarks}

This research treated an insurance market in which there are both moral hazard and insurance fraud. Our analysis derived several interesting results. First, the policyholder's effort in lowering the accident probability is a weakly monotone decreasing function of the insurance firm's investment in preventing insurance fraud. Second, the insurance firm's investment in preventing insurance fraud depends on whether it wants to provide an additional incentive to policyholder's effort in exchange for realizing the possibility of insurance fraud. Third, unlike in previous moral hazard models, the policyholder chooses a strictly positive amount of effort even in the full insurance case.

However, there are several limitations in our model. One is that the insurance contract, which represents the amounts of insurance premium, insurance money and insurance coverage rate, is taken as given. In the real world, these factors are endogenous variables of insurance firms or policyholders. If a new stage to our model to decide the insurance contract before choosing the 
amount of investment is added, we infer that both insurance firm and policyholder choose amounts of insurance premium, insurance money, and insurance coverage rate by weighing up the trade-offs between moral hazard and insurance fraud.

\section{Reference List}

Arrow, Kenneth J., 1963, Uncertainty and the Welfare Economics of Medical Care, American Economic Review, 53 (5), 941-973.

Arrow, Kenneth J., 1968, The Economics of Moral Hazard: Further Comment, American Economic Review, 58 (3), 537-539.

Baker, Tom, 1996, On the Genealogy of Moral Hazard, Texas Law Review, 75 (2), 237-292.

Boyer, Martin M., 2000a, Insurance Taxation and Insurance Fraud, Journal of Public Economic Theory, 2 (1), 101-134.

Boyer, Martin M., 2000b, Centralizing Insurance Fraud Investigation, Geneva Papers on Risk and Insurance Theory, 25 (2), 159-178.

Boyer, Martin M., 2007, Resistance (to Fraud) Is Futile, Journal of Risk and Insurance, 74 (2), 461-492.

Dionne, George, Florence Giuliano, and Pierre Picard, 2009, Optimal Auditing with Scoring: Theory and Application to Insurance Fraud, Management Science, 55 (1), 58-70.

Dorfman, Mark S., 2008, Introduction to Risk Management and Insurance (ninth edition), Pearson Prentice Hall, New Jersey.

Grossman, Sanford J., and Oliver D. Hart, 1983, An Analysis of the Principal-Agent Problem. Econometrica, 51 (1), 7-45.

Harrington, Scott E., and Gregory R. Niehaus, 2003, Risk Management and Insurance (second edition), McGraw Hill, New York.

Holmstrom, Bengt, 1979, Moral Hazard and Observability, Bell Journal of Economics, 10 (1), 74-91.

Holmstrom, Bengt, and Paul Milgrom, 1987, Aggregation and Linearity in the Provision of Intertemporal Incentives, Econometrica, 55 (2), 303-328.

Holmstrom, Bengt, and Paul Milgrom, 1991, Multitask Principal-Agent Analyses: Incentive Contracts, Asset Ownership, and Job Design, Journal of Law, Economics, and Organization, 7 (2), 24-52.

Kojima, Koji, and Mahito Okura, 2008, Effort Allocation of Insurance Agent under Asymmetric Information: An Analytical Approach, Asia-Pacific Journal of Risk and Insurance, 2 (2), 84102.

Pauly, Mark V., 1968, The Economics of Moral Hazard: Comment, American Economic Review, 58 (3), 531-537.

Pauly Mark V., 1974, Overinsurance and Public Provision of Insurance: The Roles of Moral 
Hazard and Adverse Selection, Quarterly Journal of Economics, 88 (1), 44-62.

Picard, Pierre, 1996, Auditing Claims in the Insurance Market with Fraud: the Credibility Issue, Journal of Public Economics, 63 (1), 27-56.

Powers, Michael R., 2012, Acts of God and Man: Rumination on Risk and Insurance, Columbia University Press, New York.

Rowell, David, and Luke B. Connelly, 2012, A History of the Term 'Moral Hazard', Journal of Risk and Insurance, forthcoming.

Shavell, Steven, 1979a, On Moral Hazard and Insurance, Quarterly Journal of Economics, 93 (4), 541-562.

Shavell, Steven, 1979b, Risk Sharing and Incentives in the Principal and Agent Relationship, Bell Journal of Economics, 10 (1), 55-73. 